\title{
Effects of $1 / f$ Fluctuation Music Listening on Autonomic Nervous System Activity
}

\begin{tabular}{|c|c|c|c|}
\hline Satoshi Watanabe ${ }^{* \dagger}$ & Member, & Ryouichi TAKaue ${ }^{*}$ & Non-member \\
\hline FENGHUI $\mathrm{YAO}^{\S}$ & Non-member, & Yuji Matsumoto $\mathbb{I I}$ & Non-member \\
\hline Hiroyuki Tsukamoto & Non-member, & NARUKI SHIRAHAMA** & Member \\
\hline NAOFUMI NAKAYA ${ }^{\dagger \dagger}$ & Member, & YuKIO MORI $^{\text {米 }}$ & Member \\
\hline
\end{tabular}

(Received October 11, 2017, revised March 18, 2018)

\begin{abstract}
In order to investigate the effects of listening of $1 / f$ fluctuation music, analyses of heart rate variability (HRV) by discrete Fourier transform (DFT) have been widely conducted. These methods are useful to estimate the autonomic nervous system activity (sympathetic or parasympathetic activity). To examine the effects of listening of $1 / f$ fluctuation music, three experiments are carried out. 23 healthy subjects participated the first experiment, and one piece of $1 / f$ fluctuation music is employed as the test piece. All subjects are asked to listen to the test piece individually, and their HRV are recorded and analyzed by DFT. The experiment result shows that the sympathetic nerve activity tends to decrease significantly when the subjects are listening to the test piece. The second invited 2 healthy subjects. They are asked to listen to the test piece (different from the first experiment) individually for 14 times (one time per a day), and their HRV are recorded and analyzed by DFT. The experiment result discovers linear relationship between the frequency of listening to the test piece and the changes of parasympathetic activity for both subjects. 5 healthy subjects participated the third experiment. They are asked to listen to the test piece (different from the first and second experiment) at the same time, and their HRV are recorded and analyzed by DFT. The experiment result shows that the sympathetic nerve activities of all subjects tend to decrease when they are listening to the test piece. From these results, this study concludes: (1) the effect of decreasing in sympathetic nervous activity by listening of a $1 / f$ fluctuation music is significant; (2) repeated listening of a $1 / f$ fluctuation music has effect to increase the parasympathetic activity.
\end{abstract}

Keywords: $1 / f$ fluctuation music, Heart rate variability, Sympathetic nerve activity, Parasympathetic nerve activity

\section{Introduction}

Recently, many studies about effects of listening of music have been reported. For example, Watanabe et al. investi-

\footnotetext{
* Corresponding Author: satoshi-w@iuhw.ac.jp

${ }^{\dagger}$ School of Health Sciences at Odawara, International University of Health and Welfare

1-2-25, Shiroyama, Odawara-shi, Kanagawa, 250-8588, Japan

$\$$ Graduate School of Integrated Science \& Arts, University of East Asia

2-1, Ichinomiyagakuen-cho, Shimonoseki-shi, Yamaguchi, 751-0807, Japan

$\S$ Department of Computer Science, Tennessee State University 3500 John A Merritt Blvd, Nashville, TN 37209, USA

II Junior College Division, Kindai University

3-4-1, Kowakae, Higashiosaka-shi, Osaka, 577-8502, Japan

"School of Information Studies, Shizuoka Sangyo University

4-1-1, Surugadai, Fujieda-shi, Shizuoka, 426-8668, Japan

** Department of Creative Engineering, National Institute of Technology, Kitakyushu College

5-20-1, Shii, Kokuraminami-ku, Kitakyushu-shi, Fukuoka, 802-0985, Japan

$\dagger$ Faculty of Health Sciences, Tsukuba International University 6-20-1, Manabe, Tsuchiura-shi, Ibaraki, 300-0051, Japan

$\$$ Department of Mechanical and Electronic Engineering, Salesian Polytechnic

4-6-8, Oyamagaoka, Machida-shi, Tokyo, 194-0215, Japan
}

gated the fundamental study on effects of listening of wind band music to the changes of blood pressure and heart rate in daily living environment[1]. Furthermore, in order to investigate about effects of listening of music, analyses of heart rate variability (HRV) by discrete Fourier transform (DFT) also have been conducted broadly. These methods are useful to estimate the autonomic nerve activity such as sympathetic (index of tension or excitement) or parasympathetic (index of relaxation or sedation) activity. By using these methods, Hori et al. studied the effects of music listening on HRV[2], and so on. Therefore, the effects of the music listening for HRV have been, gradually, confirmed and accepted by many people.

However, factors of the effects of listening to music on HRV are not clear. Thus, $1 / f$ fluctuation is considered as one of them[3], effects of listening of $1 / f$ fluctuation music to the changes of HRV are investigated.

The remainder of this paper is organized as follows. Section 2 describes the theory of fluctuations and HRV analysis. Experiments are shown in Section 3. Results and discussion are given in Section 4. Section 5 shows the conclusions and future works. 
Table 1: Fluctuations of the wave

\begin{tabular}{ll}
\hline$\lambda$ & Fluctuations \\
\hline 0.0 & Irregular Fluctuations \\
Close to 1.0 & $1 / f$ Fluctuation \\
$\infty$ & Regular Fluctuations \\
\hline
\end{tabular}

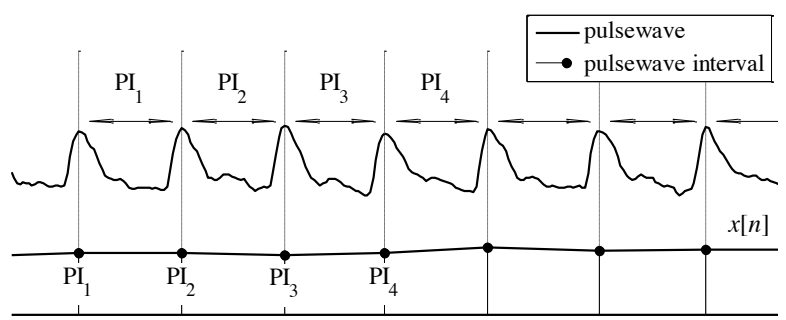

Figure 1: Pulse wave and Pulse wave interval

\section{Theory of Fluctuations and HRV Analysis}

2.1 $1 / f$ Fluctuations Fluctuation is defined as the deviation from the mean of a data. In the fluctuation, there are regular fluctuation (such as sound of the clock, predictable fluctuations) and irregular fluctuation (such as noise, unpredictable fluctuations). The fluctuations that exist between the regular fluctuation and irregular fluctuation are called $1 / f$ fluctuation[3].

$1 / f$ fluctuation is quantified as follows. A wave with fluctuation (such as music) is known to be decomposed in the frequency spectrum. In general, the spectral intensity $P$ which is inversely proportional to the frequency $f$ can be obtained according to Eq. (1) (where $\lambda$ is a constant).

$$
P=\frac{1}{f^{\lambda}}
$$

It is difficult to find $\lambda$ by directly solving Eq.(1). However, by taking the logarithm of Eq. (1), Eq. (2) is obtained, from which the value of $\lambda$ can be obtained easily.

$$
\log P=-\lambda \log f
$$

That is, the value of $\lambda$ is the value of the slope on the logarithmic plot on $P$ vs. $f$.

The fluctuations of the wave are summarized in Table 1. Note that they are determined by the value of $\lambda$, if $\lambda=1$ then the wave has a $1 / f$ fluctuation. However, if $\lambda \fallingdotseq 1$ then it is can be call that the wave has a $1 / f$ fluctuation actually.

2.2 HRV Analysis (Plus Wave Analysis) It can be regarded as synonymous with the pulse wave and heart rate analysis in healthy subjects[4]. The pulse wave can be measured easier than the heart rate. Therefore, it is possible that HRV analysis is carried out by the pulse wave analysis. In this paper, the pulse wave analysis is employed as an analysis method instead of HRV analysis.

The peak of the pulse wave obtained from the photoelectric pulse wave sensor attached to an earlobe or a finger of the subject. The pulse wave intervals (PI) are obtained from the peak of the pulse wave (see Fig. 1).

In order to proceed a pulse wave analysis from the obtained PI, it is necessary to convert them to time series data (that is, the obtained PI will be interpolated to time series data), because they are not a state of complete time series (not the isochronous intervals).

After the conversion step, the PI data which is in the time series is analyzed by the DFT. At the first step, frequency spectrum is obtained by DFT as shown in Eq. (3), the following components are extracted from them[2].

LF (Low Frequency) components: $0.04 \mathrm{~Hz}-0.15 \mathrm{~Hz}$

HF (High Frequency) components: $0.15 \mathrm{~Hz}-0.40 \mathrm{~Hz}$ Subsequently, the periodogram is applied to estimate the power spectral density (PSD) of these regions (see Eq. (4) and (5)).

$$
X[k]=\sum_{n=0}^{N-1} x[n] \mathrm{e}^{-j \frac{2 \pi k n}{N}},(k=0,1,2, \cdots, N-1)
$$

$$
\begin{aligned}
& L F=\frac{\sum\left|X_{L F}\right|^{2}}{f_{S} N}, \\
& X_{L F}=\left\{X[k] \mid 0.04 H z \leq \frac{k f_{S}}{N} \leq 0.15 H z\right\} \\
& H F=\frac{\sum\left|X_{H F}\right|^{2}}{f_{S} N}, \\
& X_{H F}=\left\{X[k] \mid 0.15 H z \leq \frac{k f_{S}}{N} \leq 0.40 H z\right\}
\end{aligned}
$$

From the calculation result of Eq. (4) and (5), autonomic nervous activity (sympathetic or parasympathetic activity) indexes can be estimated as follows[2].

$$
\begin{array}{r}
\text { Sympathetic activity index }=L F / H F \\
\text { (the ratio of (4) to (5)) }
\end{array}
$$

Parasympathetic activity index $=H F$

$$
\text { ((the value of (5)) }
$$

That is, the autonomic nervous activity is estimated by the value of Eq. (4) and (5).

According to the above preparation, the autonomic nervous activity by listening to the $1 / f$ fluctuation music can be estimated.

\section{Experiments}

To examine the effects of listening of $1 / f$ fluctuation music to the changes of HRV, three experiments are carried out.

In the first experiment, 23 healthy subjects participated, and one piece of $1 / f$ fluctuation music is employed as the test piece. All subjects are asked to listen the test piece individually, and their HRV are recorded and analyzed by DFT.

The second uses 2 healthy subjects. They are asked to listen to a test piece (different from the first experiment) individually for 14 times (one time per a day), and their HRV are recorded and analyzed by DFT.

5 healthy subjects participated in the third experiment. They are asked to listen to a test piece (different from the first and second experiment) at the same time, and their HRV are recorded and analyzed by DFT. 
Table 2: Subjects for Experiments

\begin{tabular}{ll}
\hline No. $^{* *}$ & Subjects \\
\hline$(1)$ & 23 (17 Males and 6 Females) \\
$(2)$ & 2 (Male and Female) \\
$(3)$ & 5 (2 Males and 3 Females) \\
\hline$* *$ No. is indicated experiment number
\end{tabular}

Table 3: Test Pieces (1/f fluctuation Music)

\begin{tabular}{ll}
\hline No.* $^{* *}$ & Subjects \\
\hline$(1)$ & "Requiem No.7 Lacrimosa " (Mozart) \\
$(2)$ & "Appalachian Overture” (James Barnes) \\
$(3)$ & "Sports Show March" (Yuji Koseki) \\
\hline & **No. is indicated experiment number
\end{tabular}

3.1 Subjects Three experiments are carried out in this research. The subjects involved in these experiments are summarized in Table 2.

Ethical considerations are as follows:

The explanation of this study was given by the oral and documents to the subjects, and it was also given that participation in this study was voluntary. Furthermore, the data have been collected anonymously, and have been only used carefully for the presentation of this study.

3.2 Experiment Environment The experiment is conducted in the daily living environment such as office, classroom and living room. The experiment employs the headphone (earphone) and a photoelectric pulse wave sensor attached to an earlobe or a finger of the subject. The subjects are requested to sit and do the experiment in closed-eye condition. No other special instruments is employed.

3.3 Test Pieces (1/f Fluctuation Music) Table 3 shows test pieces for the experiments. All of them have been recorded on commercial CD. Their fluctuations are ex-

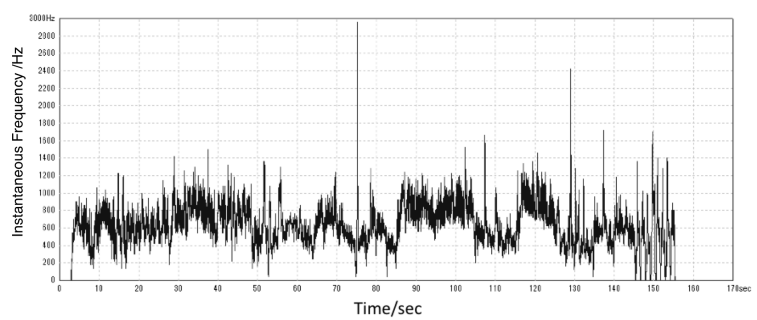

(a) "Requiem No.7 Lacrimosa" (Mozart)

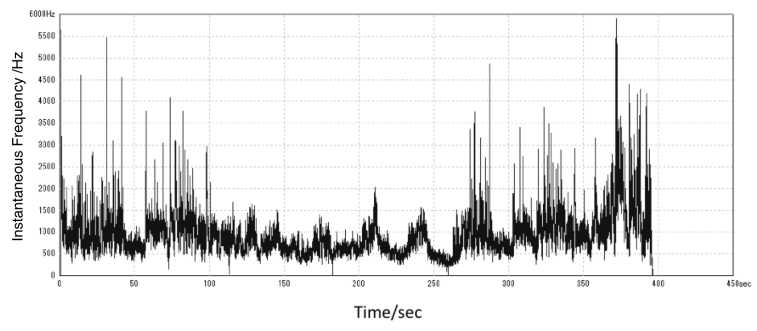

(b) “Appalachian Overture" (James Barnes)

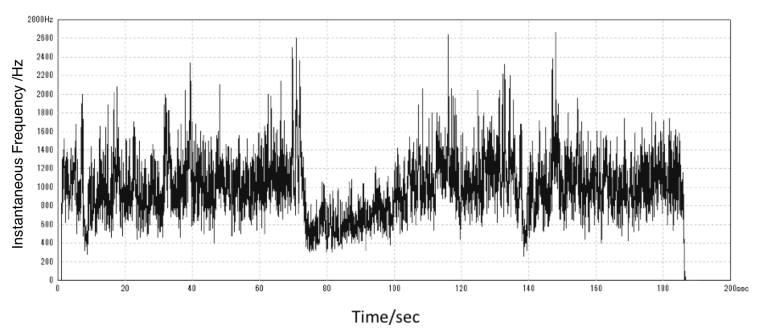

(c) "Sports Show March" (Yuji Koseki)

Figure 2: Instantaneous Frequencies of Test Pieces

amined as follows. First, the test pieces (see Table 3) are loaded into the personal computer (OS: windows) for analysis. Next, their instantaneous frequencies are obtained by zero-crossing method. Then power spectrum intensities $P$

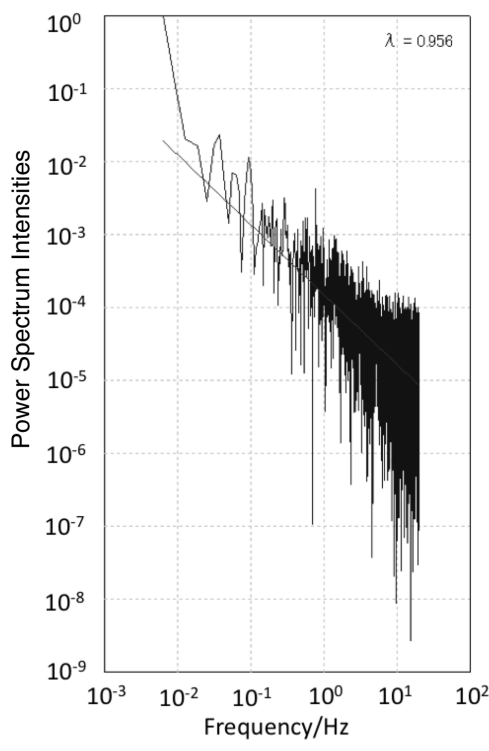

(a) "Requiem No.7 Lacrimosa" (Mozart)

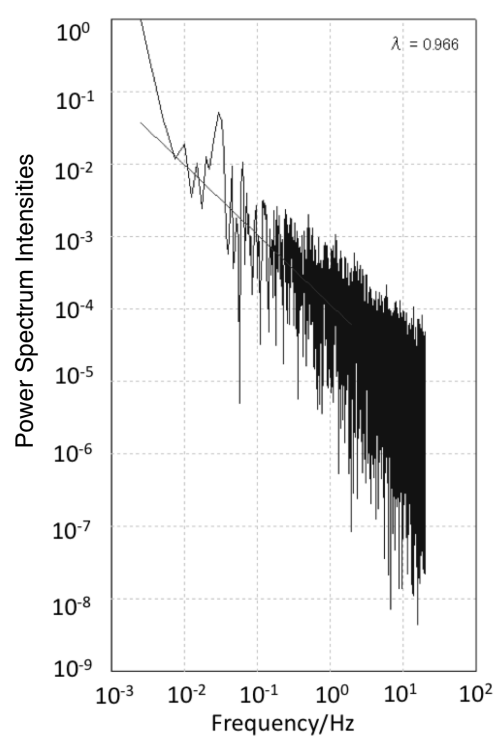

(b) "Appalachian Overture" (James Barnes)

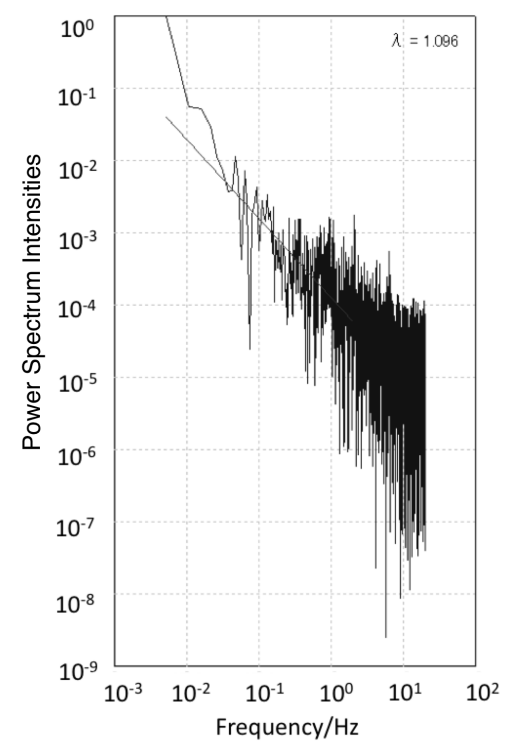

(c) "Sports Show March" (Yuji Koseki)

Figure 3: Logarithmic Plots of $P$ vs. $f$ 
Table 4: $\lambda$ value (Determination of the fluctuation)

\begin{tabular}{lll}
\hline No. $^{* *}$ & $\lambda$ value & Fluctuations \\
\hline$(1)$ & 0.956 & $1 / f$ Fluctuation \\
$(2)$ & 0.966 & $1 / f$ Fluctuation \\
$(3)$ & 1.096 & $1 / f$ Fluctuation \\
\hline **No. is indicated experiment number
\end{tabular}

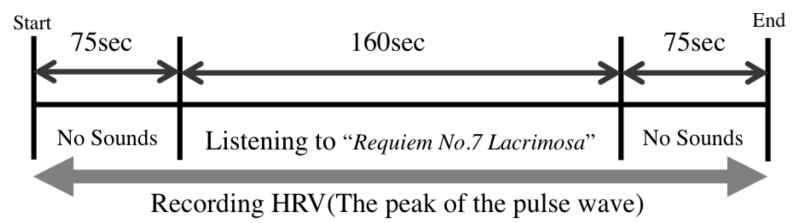

(a) Experiment (1)

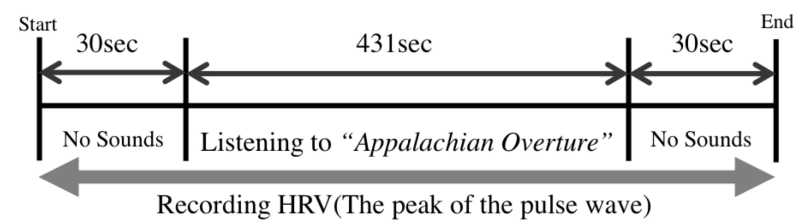

(b) Experiment (2)**

**This flow is repeated for 14 times (one time per a day)

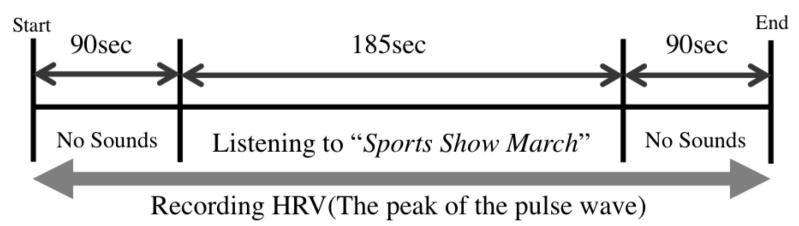

(c) Experiment (3)

Figure 4: The flow of the experiment

are determined using them. Finally, logarithmic plots of $P$ vs. frequencies $f$ are expressed. These analyzes are conducted by the software tool -Yuragi-Analyzer[5]. The results are summarized in Fig. 2 and 3.

The slope value, $\lambda$, is determined from the plot. Table 4 shows these results. From Table 4 , it is found that $\lambda$ value for all test pieces is close to 1.0. That is, it can be considered that all test pieces are $1 / f$ fluctuation music.

\subsection{Procedures of Experiments}

3.4.1 Experiment (1) One piece of $1 / f$ fluctuation music (see Table 3 ) is employed as the test piece. To play the test pieces, personal computer (OS: windows) is employed. 23 subjects are asked to listen to the test piece individually. The flow of the experiment (1) is shown in Fig. 4(a). The peak of the pulse wave obtained from the photoelectric pulse wave sensor (U-MEDICA PDUM100) attached to finger of the subject. Then, their HRV are recorded and analyzed by software (U-MEDICA "ARTETT").

3.4.2 Experiment (2) Two subjects (one male and one female) are asked to listen to the test piece (see Table 3) individually for one time per a day (total: 14times). A commercial CD player (SONY D-120) is employed to play the test pieces. The flow of the experiment (2) is shown in Fig. 4(b).

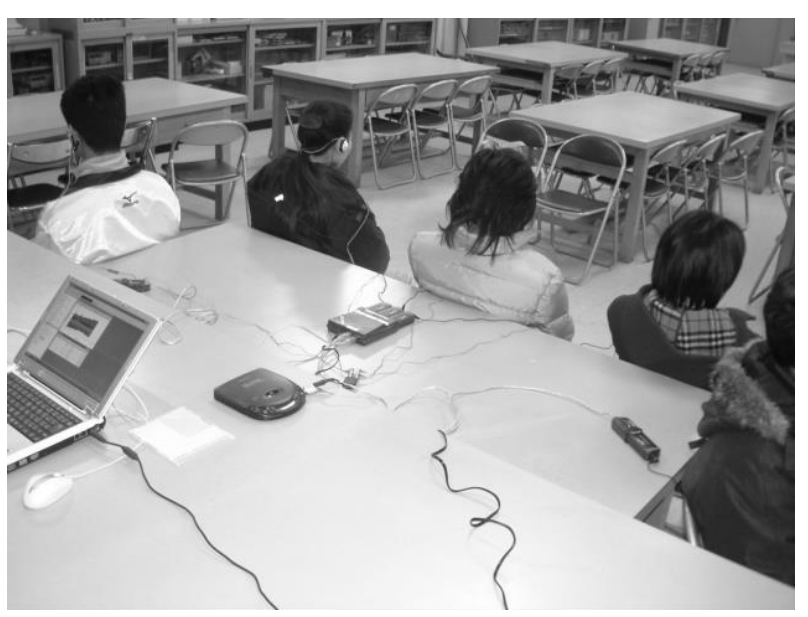

Figure 5: The Photograph of Experiment (3)

The peak of the pulse wave obtained from the photoelectric pulse wave sensor (SHIMADZU-RIKA CI6543) attached to earlobe of the subject. And their HRV are recorded and analyzed by software ("DATAStudio" (SHIMADZU-RIKA), "TSMaster" (S-TECH) and Microsoft Excel () ).

3.4.3 Experiment (3) The flow of the experiment (3) is shown in Fig. 4(c). A commercial CD player (SONY D120 ) is employed to play the test pieces. Five subjects are asked to listen to the test piece (see Table 3 ) at the same time as shown in Fig. 5.

The peak of the pulse wave obtained from the photoelectric pulse wave sensor (SHIMADZU-RIKA CI6543) attached to earlobe of the subject. And their HRV are recorded and analyzed by software ("DATAStudio" (SHIMADZU-RIKA), "TSMaster" (S-TECH) and Microsoft Excel $($ ).

\section{Results and Discussion}

4.1 Experiment (1) All subject's LF/HF indexes have been obtained from experiment (1). They are divided into three stage: before (before listening to the test piece), stimulation (listening to test piece), and after (after listening to the test piece), and their means and standard errors (SE) are calculated.

Fig. 6 shows results of these processes. When the subjects are listening to the test piece, it can be seen that the LF / HF indexes are decrease significantly (t-test, $p<0.01$ ). It can be also seen that SE is smaller at that time. This fact is the evidence that the distribution of the LF/HF index is smaller than other stage, before and after.

This is a unique result. When subjects were listening to the sounds without $1 / f$ fluctuation, changes of their $\mathrm{LF} / \mathrm{HF}$ values were not significant[6] [7]. And when subjects were listening to no sounds, their $\mathrm{LF} / \mathrm{HF}$ values hardly changed[8].

From these results and discussion, it can say that sympathetic activity is decreased significantly when the subjects are listening to $1 / f$ fluctuation music. 


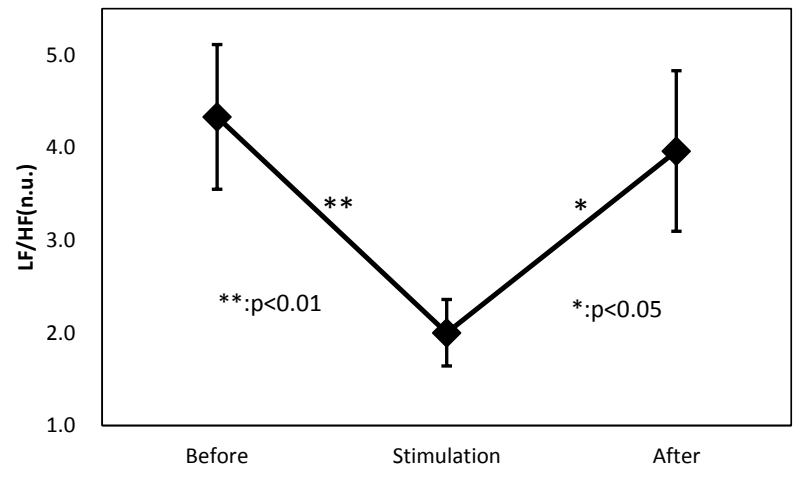

Figure 6: Changes of LF/HF indexes in Experiment (1)

४: mean \pm standard error

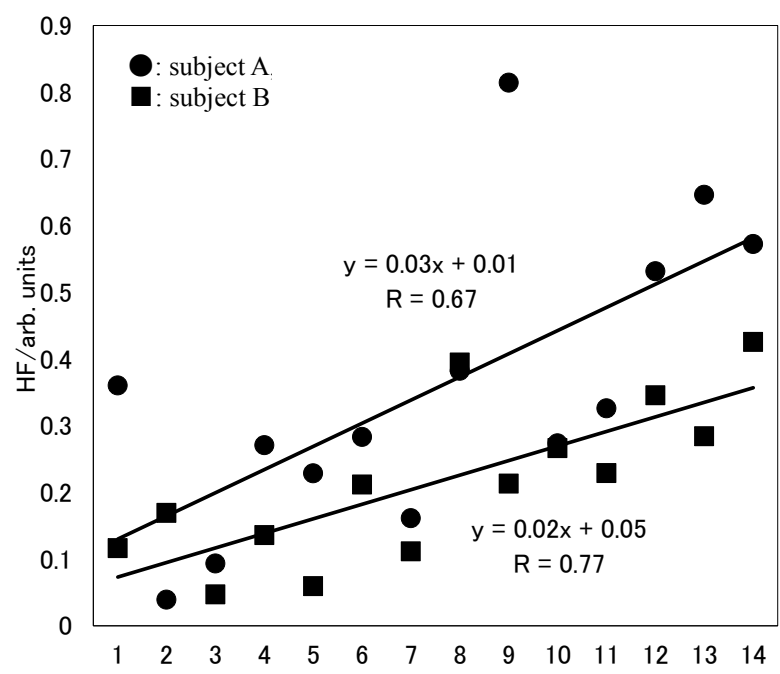

Figure 7: Changes of HF indexes in Experiment (2)

4.2 Experiment (2) In experiment (2), 2 subjects (one male and one female) are asked to listen to the test piece for 14 times (one tine per day). In other words, 14 indexes have been obtained in this experiment for each subjects. Therefore, HF indexes at the subjects are listening to the test piece in this experiment are noted, and they are plotted against listening times. These results are summarized in Fig. 7. It can be seen that a linear relationship exists between the frequency of listening to the test piece and the changes of HF indexes for both subjects.

This fact shows that parasympathetic activity is increased when the subjects are repeated listening to $1 / f$ fluctuation music.

However, it is necessary to consider whether this fact is caused by the conditions of the experiment (2), that is, the effect of repeated listening to $1 / f$ fluctuation music.

4.3 Experiment (3) Experiment (3) is carried out under the conditions of simultaneous listening to the test piece as shown in Fig. 5. 5 subject's LF/HF indexes have been obtained in this experiment. They are summarized in Fig. 8 as same as Fig. 6. When the subjects are listening test piece, it is shown that all LF/HF indexes are decreased. However, it is necessary to consider whether this effect is caused by the simultaneous listening to the test piece or not.

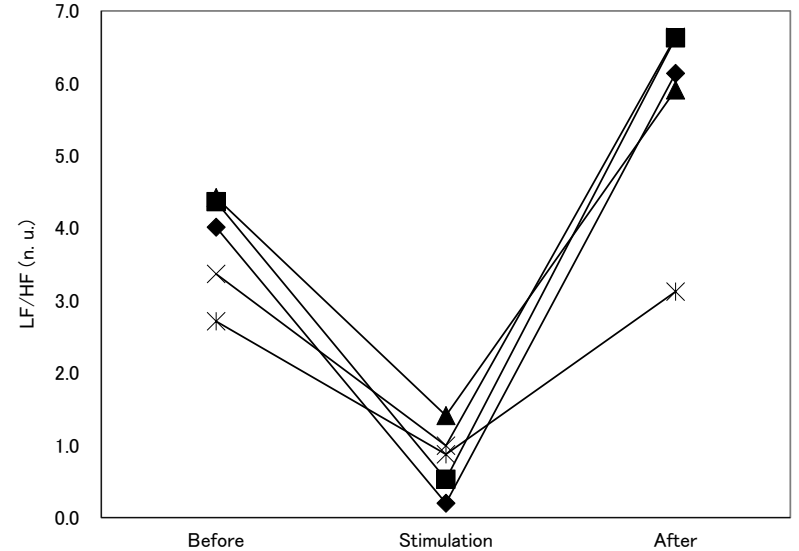

Figure 8: Changes of LF/HF indexes in Experiment (3)

From this fact and Section 4.1's results, it can say again that sympathetic activity is decreased significantly when the subjects are listening to $1 / f$ fluctuation music.

\section{Conclusions}

To examine the effects of listening of $1 / f$ fluctuation music, three experiments are carried out.

In the first experiment, the experiment result shows that the sympathetic nerve activity decreased significantly when the subjects are listening to $1 / f$ fluctuation music.

The second experiment's result discovers that a linear relationship exists between the frequency of listening to the test piece and the changes of parasympathetic activity for both subjects.

In the third experiment, the experiment result shows that the sympathetic nerve activities of all subjects decreased when they are listening to $1 / f$ fluctuation music.

From these results, this study concludes two suggestions: (1) listening of a $1 / f$ fluctuation music has a significant effect of decreasing sympathetic nervous activity; (2) repeatedly listening of a $1 / f$ fluctuation music has an effect of increasing parasympathetic activity.

However, it is necessary to consider whether these two suggestions are caused by the conditions of above experiments or not. And in order to obtain reliable conclusions, applying this method in the future study and conducting more investigation are very important.

Future works are as follows: (1) essence of the effects of listening of music, (2) variation of music, (3) essence of the effects of $1 / f$ fluctuation, (4) classifying the subjects by their preference.

\section{Acknowledgment}

This work is partially supported by JSPS Grants-in-Aid for Scientific Research, No.16K01890.

\section{References}

[1] Satoshi Watanabe, Fenghui Yao and Ryouichi Takaue, "Fundamental Study on Effects of Listening of Wind Band Music to the Changes of Blood Pressure and Heart Rate in Daily 
Living Environment”, Biomedical Soft Computing and $\mathrm{Hu}-$ man Sciences, Vol.12, No.1, pp.9-15, 2007.

[2] Kiyokazu Hori, Yasutoshi Senga, Satoshi Minami and Seiki Hori, "Effects of listening to music on heart rate variability", Japanese Journal of Biometeorology, Vol.41, No.4, pp.131$140,2004$.

[3] Toshimitsu Musha, " $1 / f$ fluctuation in biological systems", Proceedings 6th International Conference Noise in Physical System, pp.143-146, 1981.

[4] Toshiki Kikuchi, Yuji Sano, Jyunichi Abo, Shinsuke Urushidani and Tomoe Yamazaki, "Evaluation of Autonomic Nervous Activity using the Second Derivative Photoplothysmograph a-a Interval", Journal of Sport Sciences and Osteopathic Therapy, Vol.10, No.3, pp.177-183, 2009 (in Japanese).

[5] "Yuragi-Analyzer", http://mahoroba.logical-arts. $\mathrm{jp} /$ category/software/yuragi-analyzer, as of 11 October 2017 (in Japanese).

[6] Satoshi Watanabe, Masashi Agata, Kento Akitaya, Yuto Ogawa, Yuji Matsumoto, Masashi Tomita, Yuuki Kondo, Yuudai Takeuchi and Yukio Mori, "Correlation between the Subjective Evaluation by using Visual Analog Scale and Heart Rate Variability Analysis for the Effects of the Unpleasant Sound", Journal of Biomedical Fuzzy Systems Association, Vol.14, No.1, pp.19-26, 2012 (in Japanese).

[7] Satoshi Watanabe, Hiroyuki Tsukamoto, Yuji Matsumoto, Masafumi Nakagawa, Naruki Shirahama, Kazuniori Miyamoto, Naofumi Nakaya, Masashi Tomita and Yukio Mori, "Heart Rate Variability Analysis (HRV) and the Subjective Evaluation by Visual Analog Scale (VAS) for Subjects Listening to $1 / f$ Fluctuation Music and Bubbling of a Stream", Journal of Biomedical Fuzzy Systems Association, Vol.16, No.1, pp.75-84, 2014 (in Japanese).

[8] Mou Nagasaka, Michiko Tanaka, Kiyoshi Sunaga, Atsuko Masuda, Ryuya Yanagihashi, Yuka Saeki and Yoshikazu Sakakibara, "Effects of Different Sound on Heart Rate Variability", Journal of Miyazaki Prefectural Nursing University, Vol.1, No.2, pp.61-65, 2000 (in Japanese).

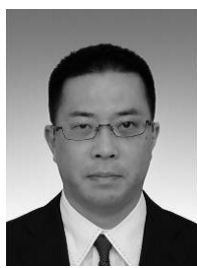

Satoshi Watanabe (Member) received his $\mathrm{Ph} . \mathrm{D}$. degree in information system from University of East Asia, Shimonoseki, in 2008. He is currently an associate professor at School of Health Sciences at Odawara, International University of Health and Welfare. His current research interests are informatics, human science and educational technology. He is a member of IIAE, BMFSA, ASJ, IPSJ, JSET, and so on.

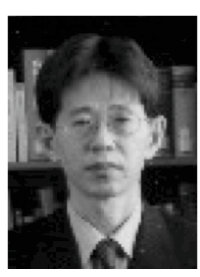

Ryouichi Takaue (Non-member) received his Doctor of Engineering degree form Kyoto University in 1988 . He is currently a professor at Graduate School of Integrated Science \& Arts, University of East Asia. His current research interests are photothermal physics, surface and interface analysis, and sensor fusion. He is a member of JSAP.

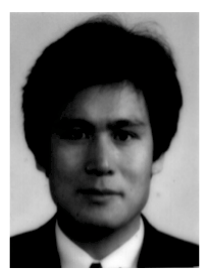

Fenghui Yao (Non-member) received his Doctor of Engineering degree from Kyushu Institute of Technology, Kitakyushu, in 1992. He joined the Department of Computer Science, Tennessee State University, as an Associate Professor, in 2004. Currently, he is a professor at Tennessee State University. He is a member of IEEE.

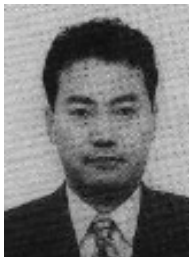

Yuji Matsumoto (Non-member) received his Ph.D. degree from Osaka University. $\mathrm{He}$ is curently a professor at Junior College Division, Kindai University. He is a member of BMFSA.

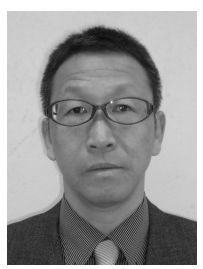

Hiroyuki Tsukamoto (Non-member) received his B.E. degree from Yokohama National University. He is curently a professor at Shizuoka Sangyo University. He is a senior coach of Japan Sports Association and Japan Volleyball Association.

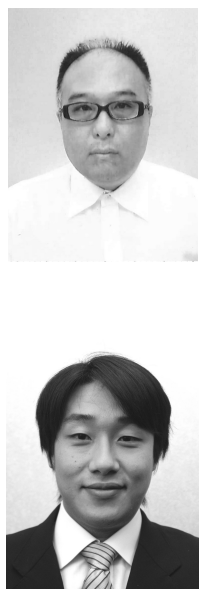

Naruki Shirahama (Member) received his $\mathrm{Ph} . \mathrm{D}$. degree in electrical engineering from Kyushu Institute of Technology in 2000. He has been working as a professor at National Institute of Technology, Kitakyushu College. His present research interests include the application of subjective observation model. $\mathrm{He}$ is a member of IIAE and BMFSA.

Naofumi Nakaya(Member) received his Ph.D. degree from Nihon University in 2015. $\mathrm{He}$ is currently an assistant professor at Tsukuba International University. $\mathrm{He}$ is a member of IIAE and BMFSA.

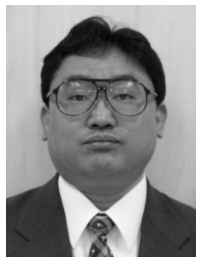

Yukio Mori (Member) received his Ph.D. degree from Tokyo University of Science. He has been working as a professor at Department of Mechanical and Electronic Engineering, Salesian Polytechnic. He is a member of IIAE and BMFSA. 